\title{
Survey results of US radiation oncology providers' contextual engagement of watch-and-wait beliefs after a complete clinical response to chemoradiation in patients with local rectal cancer
}

\author{
Jehan Yahya ${ }^{1}$, Daniel Herzig ${ }^{2}$, Matthew Farrell ${ }^{1}$, Catherine Degnin ${ }^{3}$, Yiyi Chen ${ }^{1}$, John Holland ${ }^{1}$, Simon \\ Brown $^{1}$, Christina Binder ${ }^{1}$, Jerry Jaboin ${ }^{1}$, Vassiliki Liana Tsikitis ${ }^{2}$, Nima Nabavizadeh ${ }^{1}$, Charles R. \\ Thomas Jr ${ }^{1}$, Timur Mitin ${ }^{1}$ \\ ${ }^{1}$ Department of Radiation Medicine, ${ }^{2}$ Division of Gastrointestional and General Surgery, Oregon Health and Science University, Portland, OR, \\ USA; ${ }^{3}$ Department of Biostatistics, Oregon Health and Science University Knight Cancer Institute, Portland, OR, USA \\ Contributions: (I) Conception and design: T Mitin, J Yahya; (II) Administrative support: J Holland, S Brown, C Binder, CR Thomas Jr, T Mitin, \\ J Jaboin, N Nabavizadeh; (III) Provision of study materials or patients: None; (IV) Collection and assembly of data: M Farrell, J Yahya; (V) Data \\ analysis and interpretation: C Degnin, Y Chen; (VI) Manuscript writing: All authors; (VII) Final approval of manuscript: All authors. \\ Correspondence to: Jehan Yahya, BA. Department of Radiation Medicine, Oregon Health and Science University (OHSU), 3181 S.W. Sam Jackson \\ Park Road, L337, Portland, OR, USA. Email: yahyaj@ohsu.edu.
}

Background: Watchful waiting in rectal cancer patients with a complete clinical response (cCR) to chemoradiation therapy (CRT) forgo upfront resection has been proposed. Growing evidence suggests that a watch-and-wait approach using resection for salvage of local recurrence may improve quality of life without jeopardizing outcomes. The current acceptance of watch-and-wait by US radiation oncologists (ROs) is unknown.

Methods: US ROs completed our IRB-approved anonymous e-survey regarding non-surgical management of patients who achieved a cCR to neoadjuvant CRT. Self-ranked knowledge of the OnCoRe Project-UK prospective observational study of watch-and-wait-was tested for its association with ROs' attitudes using the Chi-squared or Fisher's test, as indicated. Supporters of observation are self-identified.

Results: Of the 220 respondents, $48 \%(n=106)$ of respondents support watchful waiting and $48 \%$ claimed familiarity with the OnCoRe Project. Respondents supporting observation were more likely to be familiar with the publication $(\mathrm{P}=0.029)$. Among watch-and-wait supporters, 59\% ( $\mathrm{n}=62)$ felt comfortable discussing this approach and $41 \%$ preferred the conversation be initiated by other specialists. There was no association between comfort level in discussing watch-and-wait and familiarity with the OnCoRe Project. ROs treating more than 10 locally advanced rectal cancer (LARC) patients annually felt more comfortable discussing watch-and-wait $(\mathrm{P}=0.015)$ compared to ROs seeing fewer patients.

Conclusions: Almost half of surveyed US ROs support watch-and-wait, though many do not feel comfortable discussing this paradigm with patients. Knowledge of the OnCoRe Project is associated with support of watch-and-wait, yet not comfort level in leading the discussion. These results inform provider attitudes toward future clinical study participation.

Keywords: Rectal neoplasm; total mesorectal excision (TME); non-surgical management; multidisciplinary; radiotherapy

Submitted Jun 14, 2018. Accepted for publication Jul 23, 2018.

doi: 10.21037/jgo.2018.08.02

View this article at: http://dx.doi.org/10.21037/jgo.2018.08.02 


\section{Introduction}

The standard treatment for patients with locally advanced rectal cancer (LARC) is fluoropyrimidine-based neoadjuvant chemoradiation therapy (CRT) followed by total mesorectal excision (TME) and post-operative chemotherapy (CT) (1). Over the past decade a novel approach of omitting TME in patients who achieve a complete response to neoadjuvant CRT has been tested in the institution of University of São Paulo Medical School (2). This approach-termed watchful waiting-assumes meticulous following of patients who achieved complete response with immediate salvage surgery in the $20-30 \%$ who develop a local recurrence (3). This approach was replicated in other institutions (4) and was further supported by the OnCoRe Project, a large prospective multi-institutional propensity-score matched cohort analysis study (5).

Given the relative novelty of this approach, the lack of evidence from randomized clinical trials, and the need for multi-disciplinary expertise and commitment to this program, many physicians may not feel comfortable with recommending watchful waiting to their patients. Physicians' attitudes are likely to influence the adoption of this strategy in routine clinical practice. Current attitudes toward watchful waiting among US radiation oncologists (ROs) is unknown.

\section{Methods}

\section{Survey instrument development and data collection}

This study was approved by the Oregon Health \& Science University Institutional Review Board. We designed an online survey using REDCap software licensed by the Oregon Clinical and Translational Research Institute (OCTRI). The survey consisted of 14 questions pertaining to respondents' characteristics, non-surgical management of patients who achieved a complete clinical response (cCR) to neoadjuvant CRT and self-rated knowledge of the published OnCoRe Project. The online survey was sent anonymously by the REDCap data collecting software to 6,949 potential participants. Email invitations were sent in batches on November $16^{\text {th }}$ and $17^{\text {th }}$ of 2016 and a single reminder email was sent on November $30^{\text {th }}$, 2016.

\section{Statistical analysis}

Respondent characteristics (years in practice, practice setting, region of practice, number of rectal patients treated per year) were tested for associations with respondents' self-assessed approach to watch-and-wait in patients with rectal cancer using Chi-squared or Fisher's Exact test, as indicated. Respondents were classified as supporters of watch-and-wait approach if they marked any of the following 6 options: "Data support observation after clinical response to chemoRT, but it is not appropriate for me to start the discussion with the patient, as it should be done by a rectal surgeon", "Data support observation after clinical response to chemoRT, but I still feel uncomfortable starting this discussion myself", "Data support observation after clinical response to chemoRT, and I feel comfortable discussing this option with the patient", "If the patient asks about not doing surgery, data support observation, but I will defer the discussion to a rectal surgeon", "If the patient asks about not doing surgery, data support observation, but I feel uncomfortable having this discussion with the patient", or "If the patient asks about not doing surgery, data support observation and I feel comfortable discussing this option with the patient". Those classified as supporters of observation were further subclassified by their comfort level in leading the discussion of watch-and-wait. Respondents were identified as comfortable if they marked either of the following: "Data support observation after clinical response to chemoRT, and I feel comfortable discussing this option with the patient" or "If the patient asks about not doing surgery, data support observation and I feel comfortable discussing this option with the patient." A P value of less than 0.05 was defined as statistically significant. $R$ [version 3.3.3 (2017-03-06)] was used for all data analysis.

\section{Results}

\section{Respondent characteristics}

Many of the 6,949 email addresses in our database of potential participants were duplicates or belonged to the same physicians as these physicians are registered with both personal and institutional email accounts, making the determination of the response rate highly inaccurate. We received 337 failed/undelivered automatic responses, 7 non-applicable/ineligible responses and 220 completed responses. The characteristics of these $220 \mathrm{ROs}$ are summarized in Table 1. Of the respondents, $61 \%$ practiced over 10 years since completion of residency training, $61 \%$ work in private practice, and $55 \%$ treat 10 or fewer patients with rectal cancer per year. 
Table 1 Characteristics of 220 US RO respondents

\begin{tabular}{|c|c|}
\hline Respondent characteristics & Number of respondents $\mathrm{n}(\%)$ \\
\hline \multicolumn{2}{|l|}{$\begin{array}{l}\text { Number of years after completion } \\
\text { of residency training }\end{array}$} \\
\hline Currently in residency training & $9[4]$ \\
\hline $1-5$ & 42 [19] \\
\hline $6-10$ & 36 [16] \\
\hline$>10$ & $133[61]$ \\
\hline \multicolumn{2}{|l|}{$\begin{array}{l}\text { Number of rectal cancer patients } \\
\text { evaluated over the past } 12 \text { months }\end{array}$} \\
\hline 0 & $3[1]$ \\
\hline $1-5$ & $45[21]$ \\
\hline $6-10$ & 73 [33] \\
\hline$>10$ & $98[45]$ \\
\hline \multicolumn{2}{|l|}{ Practice setting } \\
\hline Academic center & 85 [39] \\
\hline Private practice & $135[61]$ \\
\hline \multicolumn{2}{|l|}{ Practice region } \\
\hline Northern & 34 [16] \\
\hline Pacific & 49 [22] \\
\hline Southern & $42[19]$ \\
\hline Western & $51[23]$ \\
\hline Central & $40[18]$ \\
\hline Outside US & $3[1]$ \\
\hline
\end{tabular}

Of note, one respondent failed to respond to answer questions regarding patients seen annually and region of practice. $\mathrm{RO}$, radiation oncologist.

\section{Attitudes toward watch-and-wait approach}

Of the 220 responses, $48 \%$ of respondents $(n=106)$ support the watch-and-wait approach. Forty percent claimed familiarity with the OnCoRe Project publication. Respondents supporting observation were more likely to be familiar with the OnCoRe Project (Figure 1, $\mathrm{P}=0.029$ ).

Respondents were able to select multiple responses in the survey. As such, percentages do not add up to $100 \%$. In total $48.2 \%$ of the respondents believe there is not enough data to support observation of patients who achieved a cCR in response to CRT and $12.3 \%$ are not willing to accept this approach even per patient's request (Table 2).

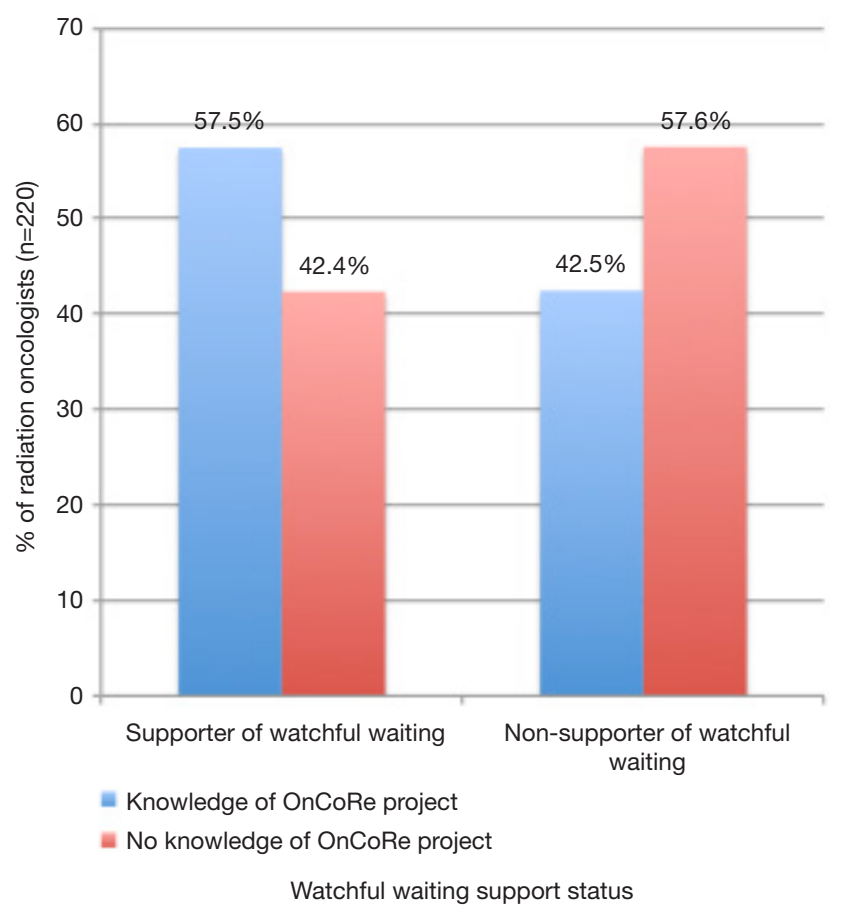

Figure 1 Association between attitudes toward watch-andwait approach in patients with a cCR to CRT and self-described familiarity with publication of the OnCoRe Project. cCR, complete clinical response; CRT, chemoradiation therapy.

\section{Comfort level in leading discussion}

Percentages do not add up to $100 \%$ as multiple reasons could be selected by respondents as listed in the previous section. Among supporters of observation, 59\% ( $\mathrm{n}=62)$ felt comfortable discussing the watch-and-wait approach and $41 \%$ preferred the conversation be initiated by other specialists. If patients are requesting to avoid surgery, $25.9 \%$ of respondents would support them, but would defer to a rectal surgeon, and $17.3 \%$ of respondents would feel comfortable leading the discussion themselves (Table 2).

A third of respondents who are familiar with OnCoRe Project report feeling comfortable discussing watchand-wait with patients. However, there is no association between knowledge of the trial and level of comfort (Table 3).

ROs treating more than 10 LARC patients annually felt more comfortable leading this discussion (Table 3, $\mathrm{P}=0.015$ ) compared to ROs seeing fewer patients. 
Table 2 Reported attitudes of respondents concerning the watch-and-wait approach

\begin{tabular}{ll}
\hline Attitudes toward observation instead of surgery & $\mathrm{n}(\%)^{*}$ \\
\hline Not enough data to support this approach & $106(48.2)$ \\
Support, but rectal surgeon should lead discussion & $29(13.2)$ \\
Support, but uncomfortable leading discussion & $30(13.6)$ \\
Support and feel comfortable leading discussion & $38(17.3)$ \\
Not acceptable, even if patient wants to avoid surgery & $27(12.3)$ \\
Support if patient wants to avoid surgery, but defer to rectal surgeon & $57(25.9)$ \\
Support if patient wants to avoid surgery, but uncomfortable leading discussion & $22(10.0)$ \\
Support if patients want to avoid surgery and feel comfortable leading discussion & $61(27.7)$ \\
\hline
\end{tabular}

*, percentages do not add up to $100 \%$ as multiple reasons could be selected by respondents.

Table 3 Associations of respondent characteristics with comfort level in discussing non-surgical approach with patients who achieved a cCR to CRT

\begin{tabular}{|c|c|c|c|}
\hline Respondent characteristics & \multicolumn{3}{|c|}{ Comfort level } \\
\hline \multicolumn{4}{|l|}{ Practice setting } \\
\hline Academic & $29(64.4)$ & $16(35.6)$ & 0.285 \\
\hline Private practice & $33(54.1)$ & $28(45.9)$ & \\
\hline 10 or fewer years & $25(62.5)$ & $15(37.5)$ & 0.514 \\
\hline$>10$ years & $37(56.1)$ & $29(43.9)$ & \\
\hline \multicolumn{4}{|c|}{ Number rectal cancer patients evaluated over a year } \\
\hline 10 or fewer patients & $24(47.1)$ & $27(52.9)$ & 0.015 \\
\hline Yes & $33(31.1)$ & $17(16.0)$ & 0.138 \\
\hline No & $29(27.4)$ & $27(25.5)$ & \\
\hline
\end{tabular}

${ }^{*}, \mathrm{P}$ value $=$ Chi-square. Significant $\mathrm{P}$ values are bolded. $\mathrm{cCR}$, complete clinical response; CRT, chemoradiation therapy.

\section{Discussion}

In 2006, Habr-Gama and colleagues, in Sao Paulo, Brazil, engineered the watch-and-wait policy after noting $26 \%$ to $38 \%$ of patients from several institutional-level series achieved a cCR after CRT $(2,6)$. In the largest series (7), 99 out of 122 patients managed under watchful waiting sustained a cCR for at least 1 year. Of the 99 patients, $13.1 \%$ had recurrences, with $5 \%$ classified as endorectal; all endorectal recurrences were salvaged. The mean recurrence interval was 52 months for local failure. Overall and disease- free 5 -year survivals were $93 \%$ and $85 \%$ (7).

Additional investigators studied watch-and-wait approach. Sanghera et al. from Birmingham, UK, found neoadjuvant CRT can lead to a cCR in up to $42 \%$ of patients in 2008 (8). In 2015, Appelt and colleagues studied watchful waiting in 55 patients with $\mathrm{T} 2$ or T3N0N1 rectal cancer treated at a Danish tertiary cancer center with high dose CRT for 6 weeks (60 Gy in 30 fractions). They reported $73 \%$ of patients achieved a cCR and $15 \%$ of patients had regrowth at one year (9).

Despite such findings, there are no randomized control 
trials investigating watch-and-wait to date probably due to logistical obstacles. This makes the OnCoRe Project all the more relevant. The OnCoRe Project is the largest propensity-score matched cohort analysis study with 259 patients either meticulously observed or operatively managed after CRT (5). This analysis found no differences in 3-year overall survival or 3-year non-regrowth diseasefree survival between watch-and-wait versus surgical resection. Patients managed under watch-and-wait had better 3-year colostomy-free survival than did those who had surgery ( $74 \%$ vs. $47 \%)$.

Our analysis revealed a dramatic polarization among US ROs regarding their views on watchful waiting. Just under half of the respondents support watchful waiting for patients with LARC who achieve a cCR after neoadjuvant CRT. Lack of adoption can at least be partially explained by almost half of our respondents citing insufficient clinical evidence for watch-and-wait. It is difficult to imagine a randomized trial in the United States for patients who achieve a cCR after neoadjuvant CT, as many patients may not choose to be randomized to immediate TME when they find out about the alternative option. Yet many organsparing treatments are done in the absence of randomized clinical evidence-such as treatment of anal cancer with the Nigro protocol (10) and bladder cancer with the trimodality approach (11). In addition, there is a lack of a single reliable tool for assessing cCR (12), and a careful follow-up of these patients is essential to ascertain that a local regrowth could be promptly salvaged with TME so as to not jeopardize patient's chance of cure.

We have limited our survey to practicing US ROs. The majority of watch-and-wait supporters felt comfortable discussing the watch-and-wait approach, with a significant portion preferring the conversation be initiated by other specialists. The fact that this conversation focuses on either omitting, deferring or undergoing surgery could explain why ROs avoid the topic. ROs who do not work in multidisciplinary teams with surgeons and medical oncologists may not feel comfortable initiating the conversation themselves. Respondents with a higher LARC patient load (more than 10 patients evaluated annually) were more likely to feel confident in initiating a conversation concerning wait-and-watch. The high-volume clinics may imply an established multidisciplinary team with a common treatment strategy.

Lack of confidence to initiate the discussion of watchful waiting after chemoradiation is not specific to ROs. Surgeons in Australasia are not confident in a conservative approach to cCR as there is a lack of reliable pathological criteria for pathologic complete response (pCR). When given a pathological interpretation of a resected specimen suggesting pCR, only $16.7 \%$ of Australasian surgeons felt comfortable equating the interpretation to no residual tumor cells in the specimen (12). Ongoing research is investigating the role of MRI and 18-fluorodeoxyglucose positron emission tomography/computed tomography (PET/CT) preoperatively to define cCR, which could help inform the decision-making process at the outset of LARC management in the future (13).

\section{Limitations}

Low response rate is our study's greatest limitation. It is likely that response bias could have influenced our results. Our survey was intended to be short and, therefore, did not capture granular information about knowledge of other trials or participation in multi-disciplinary clinics with rectal surgeons and medical oncologists.

\section{Conclusions}

This is the first analysis of contemporary views of practicing US ROs regarding a watchful waiting strategy for patients who a achieve cCR after neoadjuvant CRT. Our results suggest a dramatic polarization in practitioners' attitudes, and amongst those who support this treatment paradigm, there was much division in the levels of comfort leading this discussion.

These results suggest a general equipoise toward watchful waiting on the level of practicing US ROs and are important for the design of future prospective clinical trials.

\section{Acknowledgments}

We thank the respondents who have taken the time to participate and complete our survey.

Funding: This work was support by OHSU REDCap (1 UL1 RR024140 01).

\section{Footnote}

Conflicts of Interest: The authors have no conflicts of interest to declare.

Ethical Statement: The study was approved by institutional review board of Oregon Health and Science University (IRB 
protocol 11149), and the requirement to obtain informed consent from survey respondents was waived.

\section{References}

1. Lynn PB, Strombom P, Garcia-Aguilar J. OrganPreserving Strategies for the Management of NearComplete Responses in Rectal Cancer after Neoadjuvant Chemoradiation. Clin Colon Rectal Surg 2017;30:395-403.

2. Habr-Gama A, Perez RO, Nadalin W, et al. Operative versus nonoperative treatment for stage 0 distal rectal cancer following chemoradiation therapy: long-term results. Ann Surg 2004;240:711-7; discussion 717-8.

3. Yang TJ, Goodman KA. Predicting complete response: is there a role for non-operative management of rectal cancer? J Gastrointest Oncol 2015;6:241-6.

4. Smith JJ, Chow OS, Gollub MJ, et al. Organ Preservation in Rectal Adenocarcinoma: a phase II randomized controlled trial evaluating 3 -year disease-free survival in patients with locally advanced rectal cancer treated with chemoradiation plus induction or consolidation chemotherapy, and total mesorectal excision or nonoperative management. BMC Cancer 2015;15:767.

5. Renehan AG, Malcomson L, Emsley R, et al. Watchand-wait approach versus surgical resection after chemoradiotherapy for patients with rectal cancer (the OnCoRe project): a propensity-score matched cohort analysis. Lancet Oncol 2016;17:174-83.

6. Habr-Gama A, Sabbaga J, Gama-Rodrigues J, et al. Watch and wait approach following extended neoadjuvant chemoradiation for distal rectal cancer: are we getting

Cite this article as: Yahya J, Herzig D, Farrell M, Degnin C, Chen Y, Holland J, Brown S, Binder C, Jaboin J, Tsikitis VL, Nabavizadeh N, Thomas CR Jr, Mitin T. Survey results of US radiation oncology providers' contextual engagement of watch-and-wait beliefs after a complete clinical response to chemoradiation in patients with local rectal cancer. J Gastrointest Oncol 2018;9(6):1127-1132. doi: 10.21037/ jgo.2018.08.02 closer to anal cancer management? Dis Colon Rectum 2013;56:1109-17.

7. Habr-Gama A, Perez RO, Proscurshim I, et al. Patterns of failure and survival for nonoperative treatment of stage $\mathrm{c} 0$ distal rectal cancer following neoadjuvant chemoradiation therapy. J Gastrointest Surg 2006;10:1319-28; discussion 1328-9.

8. Sanghera P, Wong DW, McConkey CC, et al. Chemoradiotherapy for rectal cancer: an updated analysis of factors affecting pathological response. Clin Oncol (R Coll Radiol) 2008;20:176-83 .

9. Appelt AL, Pløen J, Harling H, et al. High-dose chemoradiotherapy and watchful waiting for distal rectal cancer: a prospective observational study. Lancet Oncol 2015;16:919-27.

10. Leichman L, Nigro N, Vaitkevicius VK, et al. Cancer of the anal canal. Model for preoperative adjuvant combined modality therapy. Am J Med 1985;78:211-5.

11. Mitin T. Radical Cystectomy is the best choice for most patients with muscle-invasive bladder cancer? Opinion: No. Int Braz J Urol 2017;43:188-91.

12. Behrenbruch C, Ryan J, Lynch C, et al. Complete clinical response to neoadjuvant chemoradiotherapy for rectal cancer: an Australasian perspective. ANZ J Surg 2015;85:103-4.

13. Fischkoff KN, Ruby JA, Guillem JG. Nonoperative approach to locally advanced rectal cancer after neoadjuvant combined modality therapy: challenges and opportunities from a surgical perspective. Clin Colorectal Cancer 2011;10:291-7. 